\title{
To Calculation of Rectangular Plates on Periodic Oscillations
}

\author{
Rustamkhan Abdikarimov ${ }^{1}$, Dadakhan Khodzhaev ${ }^{2, *}$, and Nikolay Vatin ${ }^{3}$ \\ ${ }^{1}$ Tashkent Financial Institute, A. Temur St. 60A, Tashkent, 100000, Uzbekistan \\ ${ }^{2}$ Tashkent Institute of Irrigation and Agricultural Mechanization Engineers, Kari Niyazov St. 39, \\ Tashkent, 100000, Uzbekistan \\ ${ }^{3}$ Peter the Great St. Petersburg Polytechnic University, Polytechnicehskaya 29, St. Petersburg, \\ 195251, Russian Federation
}

\begin{abstract}
Geometrically nonlinear mathematical model of the problem of parametric oscillations of a viscoelastic orthotropic plate of variable thickness is developed using the classical Kirchhoff-Love hypothesis. The technique of the nonlinear problem solution by applying the BubnovGalerkin method at polynomial approximation of displacements (and deflection) and a numerical method that uses quadrature formula are proposed. The Koltunov-Rzhanitsyn kernel with three different rheological parameters is chosen as a weakly singular kernel. Parametric oscillations of viscoelastic orthotropic plates of variable thickness under the effect of an external load are investigated. The effect on the domain of dynamic instability of geometric nonlinearity, viscoelastic properties of material, as well as other physical-mechanical and geometric parameters and factors are taken into account. The results obtained are in good agreement with the results and data of other authors.
\end{abstract}

\section{Introduction}

Construction of the dynamic instability domain of thin-walled elastic systems is a very urgent and important task; a multitude of publications are devoted to this issue, of which, first of all, the studies carried out in [1-6] should be mentioned. Beginning with the article by N.M. Belyaev [1], parametric oscillations become the subject of numerous studies in application to various mechanical systems with distributed parameters, in particular, to rods, plates and shells. In the fundamental monograph V.V. Bolotin [2] has analyzed in detail the occurrence of parametric resonance in elastic thin-walled systems. An extensive monograph by $\mathrm{T}$. Schmidt [6] contains a detailed review of many aspects concerning these issues.

Solution of linear problems of parametric oscillations of thin-walled structures (such as plates and shells) often is reduced to the use of ordinary differential equations with periodic coefficients - to the Mathieu-Hill equations. At certain ratios of coefficients, the solution of this type of equation increases indefinitely, since the corresponding oscillations of thinwalled structures are resonant in nature. The range of parameters that cause these oscillations is called the domain of dynamic instability (DDI). An assessment of the DDI is one of the central problems of parametric oscillations of thin-walled structures.

\footnotetext{
*Corresponding author: dhodjaev@mail.ru
} 
Solution of the problems connected with the study of resonant phenomena in geometrically nonlinear cases of viscoelastic thin-walled structures of variable thickness has been developed inadequately. The development of a methodology for solving such an important class of problems is of considerable theoretical and practical interest.

Note the following important publications in recent years devoted to the solution of this scientific problem:

- in the article by Kurpa L., Mazur O.S., Tkachenko Ya.V. [7] a numerical-analytical method for studying parametric oscillations of plates under the effect of static and dynamic loads (bearing a periodic nature) is substantiated;

- in the article by I.D. Ievzerov [8] the stability problems for bars and plates are considered. Variation formulations are used for the stability problem;

- the article by V.M. Dubrovin and T.A. Butina [9]; the authors propose a method for calculating dynamic stability of a cylindrical shell under axial compressive dynamic load;

- in the article by A.A. Mochalin [10] on the basis of the V.Z. Vlasov semi-momentless theory, the solution of the problem of dynamic stability of an isotropic cylindrical shell of variable thickness is considered in the direction of its generatrix under external pressure;

- in the article [11] (Usarov M.K.) is dedicated to improvement of plate theory in order to take into account forces, moments and bimoments, generated by nonlinear law of displacement distribution in plate cross-sections;

- the article [12] (Dey T., Ramachandra L.S.) is devoted to parametric oscillations of shells (of various types) subjected to static and dynamic loads of periodic nature (equations of oscillatory motion are obtained by the authors using the Donnell's theory of shell);

- in the article [13] the author Budanov V.M. has proposed a special linear transformation for expressing the general solution of a second-order differential equation with periodic coefficients; the author has shown with numerical experiments that there exist the periodic solutions of the auxiliary system outside the instability domains of solutions of the Mathieu equation, and the solutions obtained for the DDI are in good agreement with known results;

- in the articles [14] (Abdikarimov R.A., Goloskokov D.P.) and [15] (Abdikarimov R.A., Khudayarov B.A.) a problem of nonlinear fluctuations of a viscoelastic plate of a variable thickness is considered. The resolving system of the integrodifferential equations of a problem is received. The numerical decision is constructed by Bubnov-Galerkins method with the subsequent application of quadrature formulas for approximation of integrals;

- in the article [16] (Eshmatov B.Kh., Eshmatov Kh., Khodzhaev D.A.) the problem of flutter of viscoelastic rectangular plates and cylindrical panels with concentrated masses is studied in a geometrically nonlinear formulation. The behavior of viscoelastic rectangular plates and cylindrical panels is studied and the critical flow velocities are determined for real composite materials over wide ranges of physicomechanical and geometrical parameters.

Practical value of studies on the assessment of stability of thin-walled structures consists in consideration of the magnitude of existing loads and the law of their variation over time, as well as of all important features of both the structure itself and the properties of materials for their fabrication; this allows a correct estimation of load-bearing capacity of such structures.

Despite the great number of publications devoted to the study of parametric oscillations of thin-walled structures, the number of papers devoted to the study of dynamic stability of these structures with account of viscoelastic properties of their material is few. 


\section{Methods}

Consider a viscoelastic rectangular plate (with sides $a$ and $b$ ) of variable thickness $h=h(x, y)$, made of homogeneous isotropic material (Fig. 1). Assume that the plate is subjected to an external dynamic load acting along the edge $a$, having a periodic character: $P(t)=P_{0}+P_{1} \cos \Theta t$ (here $P_{0}, P_{1}=$ const $; \Theta-$ is the excitation frequency). Also assume that the plate has initial deflections.

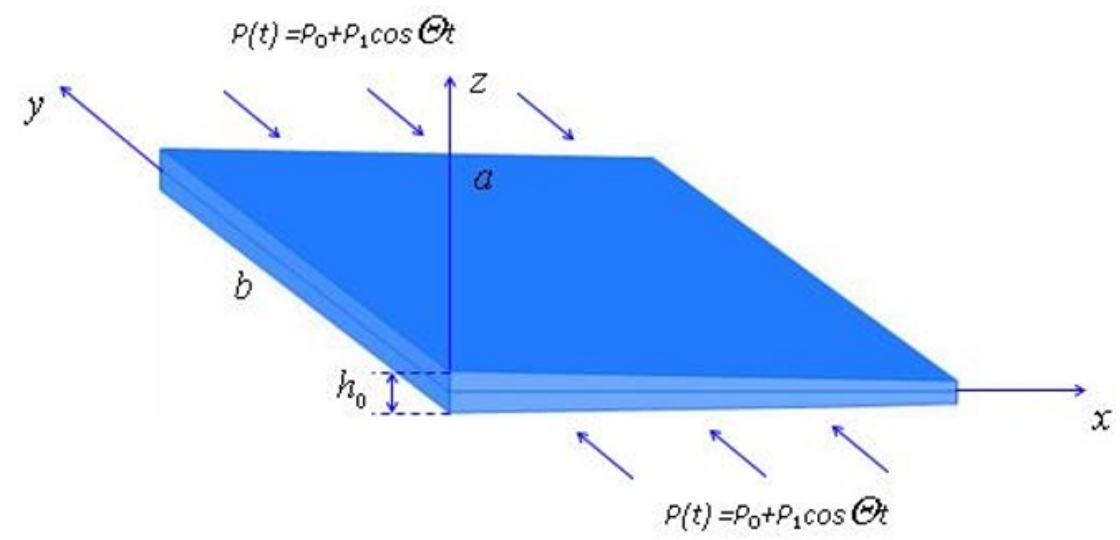

Fig.1.

In [17] R.A. Abdikarimov and V.M. Zhgutov have shown that in this case nonlinear equations of the oscillatory motion of the plate (with respect to deflection $w=w(x, y, t)$ and longitudinal displacements $u=u(x, y, t), v=v(x, y, t))$ under the influence of force $P(t) \frac{\partial^{2} w}{\partial x^{2}}$ with account of initial deflection (at appropriate boundary and initial conditions) can be written in the form:

$$
\begin{gathered}
\left(1-\Gamma^{*}\right)\left[h\left(\frac{\partial \varepsilon_{x}}{\partial x}+\mu \frac{\partial \varepsilon_{y}}{\partial x}+\frac{1-\mu}{2} \frac{\partial \gamma_{x y}}{\partial y}\right)+\frac{\partial h}{\partial x}\left(\varepsilon_{x}+\mu \varepsilon_{y}\right)+\frac{1-\mu}{2} \frac{\partial h}{\partial y} \gamma_{x y}\right]+ \\
+\frac{1-\mu^{2}}{E} p_{x}-\rho h \frac{1-\mu^{2}}{E} \frac{\partial^{2} u}{\partial t^{2}}=0 \\
\left(1-\Gamma^{*}\right)\left[h\left(\frac{\partial \varepsilon_{y}}{\partial y}+\mu \frac{\partial \varepsilon_{x}}{\partial y}+\frac{1-\mu}{2} \frac{\partial \gamma_{x y}}{\partial x}\right)+\frac{\partial h}{\partial y}\left(\varepsilon_{y}+\mu \varepsilon_{x}\right)+\frac{1-\mu}{2} \frac{\partial h}{\partial x} \gamma_{x y}\right]+ \\
+\frac{1-\mu^{2}}{E} p_{y}-\rho h \frac{1-\mu^{2}}{E} \frac{\partial^{2} v}{\partial t^{2}}=0 \\
\left(1-\Gamma^{*}\right)\left\{\left[D \nabla^{4} w+2 \frac{\partial D}{\partial x} \frac{\partial}{\partial x} \nabla^{2} w+2 \frac{\partial D}{\partial y} \frac{\partial}{\partial y} \nabla^{2} w+\nabla^{2} D \nabla^{2} w-\right.\right. \\
\left.\left.-(1-\mu)\left(\frac{\partial^{2} D}{\partial x^{2}} \frac{\partial^{2} w}{\partial y^{2}}-2 \frac{\partial^{2} D}{\partial x \partial y} \frac{\partial^{2} w}{\partial x \partial y}+\frac{\partial^{2} D}{\partial y^{2}} \frac{\partial^{2} w}{\partial x^{2}}\right)\right]\right\}+
\end{gathered}
$$




$$
\begin{gathered}
+h\left[\frac{\partial}{\partial x}\left(\frac{\partial w}{\partial x} \sigma_{x}+\frac{\partial w}{\partial y} \tau_{x y}\right)+\frac{\partial}{\partial y}\left(\frac{\partial w}{\partial y} \sigma_{y}+\frac{\partial w}{\partial x} \tau_{x y}\right)\right]+\frac{\partial h}{\partial x}\left(\frac{\partial w}{\partial x} \sigma_{x}+\frac{\partial w}{\partial y} \tau_{x y}\right)+ \\
+\frac{\partial h}{\partial y}\left(\frac{\partial w}{\partial y} \sigma_{y}+\frac{\partial w}{\partial x} \tau_{x y}\right)+P(t) \frac{\partial^{2} w}{\partial x^{2}}+q-\rho h \frac{\partial^{2} w}{\partial t^{2}}=0
\end{gathered}
$$

Introduce to equations (1) the dimensionless parameters and using previous notations, a system of integral-differential equations (IDE) in a dimensionless form is obtained.

To solve the system of nonlinear IDE (1), the sought for functions $u(x, y, t), v(x, y, t)$, $w(x, y, t), w_{0}(x, y, t)$ are presented as an expansion in known (basic) functions $\phi_{n m}(x, y)$, $\varphi_{n m}(x, y), \psi_{n m}(x, y), \psi_{0 n m}(x, y)$ satisfying the given boundary conditions:

$$
\begin{aligned}
u(x, y, t) & =\sum_{n=1}^{N} \sum_{m=1}^{M} u_{n m}(t) \phi_{n m}(x, y), v(x, y, t)=\sum_{n=1}^{N} \sum_{m=1}^{M} v_{n m}(t) \varphi_{n m}(x, y), \\
w(x, y, t) & =\sum_{n=1}^{N} \sum_{m=1}^{M} w_{n m}(t) \psi_{n m}(x, y), w_{0}(x, y)=\sum_{n=1}^{N} \sum_{m=1}^{M} w_{0 n m} \psi_{0 n m}(x, y)
\end{aligned}
$$

where $u_{n m}=u_{n m}(t), v_{n m}=v_{n m}(t), \quad w_{n m}=w_{n m}(t)$ - are unknown time functions; $\phi_{n m}(x, y), \varphi_{n m}(x, y), \psi_{n m}(x, y), \psi_{0 n m}(x, y), n=1,2, \ldots, N ; m=1,2, \ldots, M$ - are known (basic) coordinate functions that satisfy the given boundary conditions of the problem (described, for example, in Volmir's monograph [4]).

As a rule, algorithms for solving dynamic problems for viscoelastic thin-walled structures are standard ones. By choosing the basic functions that satisfy certain boundary conditions, the original boundary value problem is reduced to the problem of oscillations of a system with a finite number of degrees of freedom, i.e. to a system of linear (or nonlinear) IDE with one independent variable (of time). As a rule, trigonometric functions or beam functions are used as basic ones. It is known that the choice of such basic functions in many cases limits the class of solved problems to structures of the simplest configurations beams of constant sections, rectangular plates, cylindrical shells [4].

For elastic structures of a more complex geometric form, it is very tempting to use as basic functions $\phi_{n m}(x, y), \varphi_{n m}(x, y), \psi_{n m}(x, y), \quad \psi_{0 n m}(x, y)$ the eigenmodes of oscillations, which are, in a sense, "native" and allow better consideration of all the features of these structures. For the first time, the expansion of the solution in terms of eigenmodes of oscillations in solving problems of described class was used in [18] (by Chelomei V.N.). Subsequently, the eigenmodes of oscillations in the process of expansion of the sought for solution in the problem of forced oscillations of real elastic and viscoelastic structures (including the shell type) were used in [19] (Ishmatov A.N., Mirsaidov M.M.) and [20] (Sultanov T.Z., Khodzhaev D.A., Mirsaidov M.M.).

In [21] (Mirsaidov M.M., Mekhmonov Ya.), along with expansion in terms of eigenmodes of oscillations, the problem of vibrations of three-dimensional bodies with associated mass was solved. As is well known, the determination of eigenmodes of oscillations for any structures is a completely independent and a very difficult task. That is why quite often a part of a complete system of known functions in analytical form, that satisfies a particular boundary value problem, is used as a basic function.

Substituting (2) into the system (1) written in dimensionless parameters, and following the Bubnov-Galerkin procedure for the functions $u_{n m}(t), v_{n m}(t), w_{n m}(t)$ to be determined, a system of nonlinear IDE, i.e. the basic resolving nonlinear IDE are obtained.

Twice integrating the obtained system with respect to time $t$, it can be written in 
integral form, as shown in the articles by A.F. Verlan, R.A. Abdikarimov, H. Eshmatov [22].

\section{Results and Discussion}

Results of computational experiments corresponding to different physical and geometric parameters of a viscoelastic isotropic plate are illustrated by the graphs shown in Figs. 2-3. The dependence of the change in thickness is taken in the form

$$
h(x)=\frac{1}{2} h_{0}\left(1-\alpha^{*} x\right) .
$$

Fig. 2 shows the time $t$ variation of displacements of the midpoint $(x=0.5, y=0.5)$ for different values of rheological parameter $A$ of the relaxation kernel of material. An analysis of results has shown that in all the cases considered, at initial time, the oscillations occur near the equilibrium (initial) state. Over time, the amplitude slowly increases and reaches the value comparable with the one of plate thickness. Curve $1(A=0)$ corresponds to an elastic solution (without considering viscoelastic properties of material); here the oscillations occur without attenuation. Note that in this case the amplitude of oscillations does not increase, but the beating occurs, which is explained by the proximity of the frequency of natural oscillations of the plate to the frequency of periodic effects. Curve 2 ( $A=0.05)$ and curve $3(A=0.1)$ are obtained considering the viscoelastic properties of material. It is seen that damped oscillations occur near initial oscillations, but over time, oscillations occur with practically constant amplitude, i.e. become steady. In this case, there is some deviation of oscillations from the neutral axis, which is explained by the presence of initial imperfections in the shape.

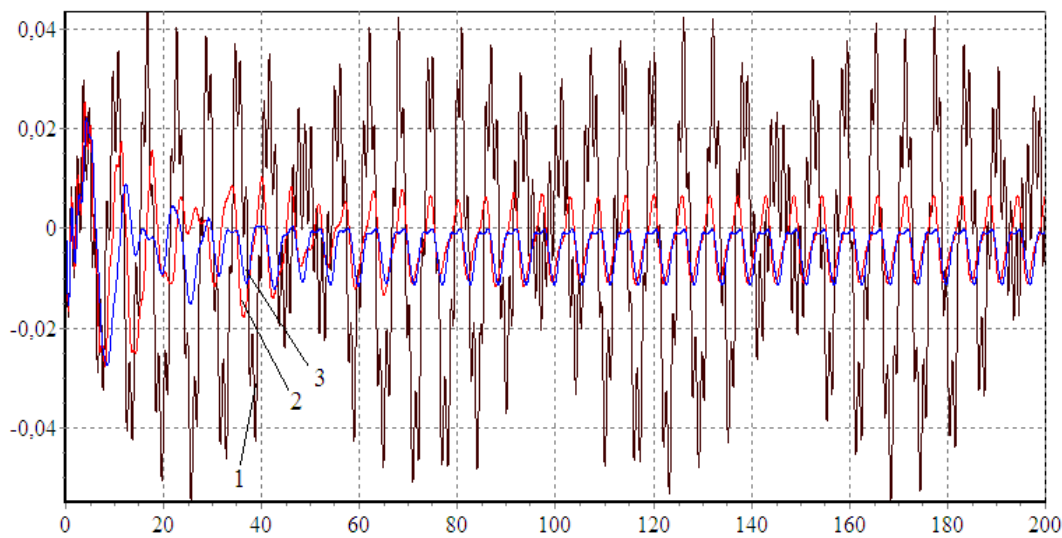

Fig. 2. Graphs of variation with time $t$ of displacements of the midpoint $(x=0.5, y=0.5)$ of the plate at different values of the relaxation kernel parameter $A$ :

$$
\begin{aligned}
& \alpha=0.25 ; \beta=0.05 ; \mu=0.3 ; \delta=25 ; w_{0}=0.01 ; q=0 ; \lambda=1 ; \alpha^{*}=0.5 ; \delta_{0}=0 ; \delta_{1}=0.5 \\
& ; \Theta_{1}=1.1 ; A=0.0(1) ; 0.05(2) ; 0.1(3)
\end{aligned}
$$

Figure 3 shows the graphs of deflection versus time $t$ for the midpoint ( $x=0.5, y=0.5$ ) of the plate at various values of its thickness parameter $\alpha^{*}$, taking into account viscoelastic properties of material. Namely: at $\alpha^{*}=0$ (curve 1); at 0.4 (curve 2); 
at 0.8 (curve 3). Note that an increase in the parameter of $\alpha^{*}$ according to the above law corresponds to a decrease in thickness of the plate along the length of $O x$ axis. This causes the resonance regime and an increase in oscillation amplitude of the plate (curve 3). At other values of parameter $\alpha^{*}$ (curves 1 and 2), the oscillations become steady.

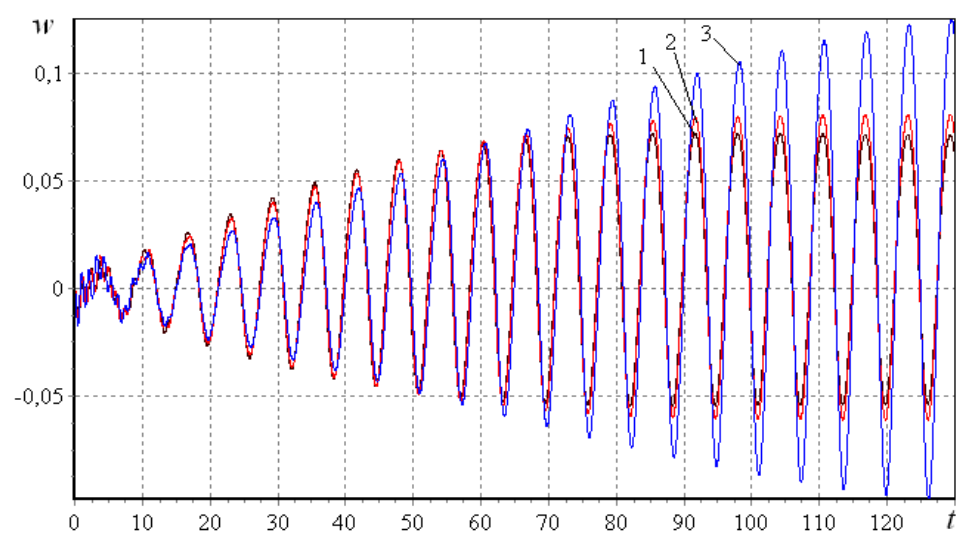

Fig. 3. Graphs of dependence of deflection on time $t$ of the midpoint $(x=0.5, y=0.5)$ of the plate at various values of the parameter of its thickness $\alpha^{*}$ :

$A=0.05 ; \alpha=0.25 ; \beta=0.05 ; \mu=0.3 ; \delta=25 ; \lambda=1 ; w_{0}=0.01 ; q=0 ; \delta_{0}=0 ; \delta_{1}=0.5$;

$\Theta_{1}=1.1 ; \alpha^{*}=0(1) ; 0.4(2) ; 0.8(3)$

\section{Conclusions}

1. Mathematical model, technique for its investigation and computational algorithm for the problem of parametric nonlinear oscillations of plates of variable thickness are developed taking into account viscoelastic properties of material.

2. An appropriate computer software package is designed to effectively evaluate dynamic instability of plates in question, depending on mechanical characteristics of their material, external excitation loads, geometric parameters, eigenfrequency of vibration, and excitation forces.

As a result of numerical experiments it was established that:

1) the presence of viscoelastic properties of material leads to a steady-state oscillation regime even in resonance modes;

2) the presence of initial imperfections of the form leads to a deviation from the reference plane, which is assumed to be neutral (due to the deviation of the latter);

3) a decrease in thickness of the plate along its length in some cases can lead to a resonance mode of oscillations.

Besides, the dependences of some parameters of stable oscillatory process of the plates on the amplitude and frequency of external transverse load are obtained.

\section{References}

1. N.M. Belyaev, Engineering Constructions and Structural Mechanics, 149-167 (1924)

2. V.V. Bolotin, Dynamic Stability of Elastic Systems, Gostekhizdat, Moscow, (1956) 
3. V.Z. Vlasov, General Theory of Shells and its Applications in Engineering, Gostekhizdat, Moscow-Leningrad, (1949)

4. A.S. Volmir, Nonlinear Dynamics of Plates and Shells (Science, Moscow, 1972)

5. A.E. Bogdanovich, Nonlinear Problems of the Dynamics of Cylindrical Composite Shells (Zinatne, Riga, 1987)

6. T. Schmidt, Parametric Oscillations (Mir, Moscow, 1978)

7. L. Kurpa, O.S. Mazur, Ya.V. Tkachenko, J. Math. Sci., 203 (2), 165-184 (2014)

8. I.D. Ievzerov, Mag. Civ. Eng., 1, 6-11 (2014)

9. V.M. Dubrovin, T.A. Butina, Mathematical Modeling and Computational Methods, 3 (11), 24-32 (2016)

10. A.A. Mochalin, Izv. Saratov Univ. Ser. Math. Mech. Inform, 15 (2), 210-215 (2015)

11. M.K. Usarov, Mag. Civ. Eng., 1, 80-90 (2015)

12. T. Dey, L.S. Ramachandra, J. Sound Vib., 353, 272-291 (2015)

13. V.M. Budanov, Moscow University Mechanics Bulletin, 72 (1), 7-12 (2017)

14. R.A. Abdikarimov, D.P. Goloskokov, Journal of University of Water Communications, 2 (9), 102-107 (2011)

15. R.A. Abdikarimov, B.A. Khudayarov, Mechanics. Proceedings of National Academy of Sciences of Armenia, 4 (64), 30-38 (2011)

16. B.Kh. Eshmatov, Kh. Eshmatov, D.A. Khodzhaev, J. Appl. Mech. Tech. Phys., 4 (54) 578-587 (2013)

17. R.A. Abdikarimov, V.M. Zhgutov, Mag. Civ. Eng., 6, 38-47 (2010)

18. V.N. Chelomei, Dynamic Stability of the Elements of Aircraft Structures (Aeroflot, Moscow, 80 (1939)

19. A.N. Ishmatov, M. Mirsaidov, Soviet Applied Mechanics, 27 (4), 68-74 (1991)

20. T.Z. Sultanov, D.A. Khodzhaev, M.M. Mirsaidov, Mag. Civ. Eng., 1 (45), 80-89 (2014)

21. M. Mirsaidov, Ya. Mekhmonov, J. Strength of Materials, 3, 111-116 (1987)

22. A.F. Verlan, R.A. Abdikarimov, H. Eshmatov, Electronic modeling, 32 (2), 3-14 (2010) 